\title{
Deregulation of microRNA-31a-5p is involved in the development of primary hypertension by suppressing apoptosis of pulmonary artery smooth muscle cells via targeting TP53
}

\author{
QIANG FENG ${ }^{1}$, TAO TIAN $^{2}$, JUNFENG LIU ${ }^{3}$, LI ZHANG $^{4}$, JIANGANG QI ${ }^{5}$ and XIAOJUAN LIN ${ }^{6}$ \\ ${ }^{1}$ Department of Laboratory, The People's Hospital of Tongchuan, Tongchuan, Shaanxi 727000; \\ ${ }^{2}$ Department of Laboratory, Second Affiliated Hospital of Shaanxi Chinese Traditional Medicine, Xianyang, Shaanxi 712000; \\ Departments of ${ }^{3}$ Infection and ${ }^{4}$ Gynecology and Obstetrics, The People's Hospital of Tongchuan; \\ ${ }^{5}$ Department of Laboratory, Tongchuan Hospital of Chinese Traditional Medicine; \\ ${ }^{6}$ Department of Cardiology, The People's Hospital of Tongchuan, Tongchuan, Shaanxi 727000, P.R. China
}

Received October 17, 2017; Accepted March 9, 2018

DOI: $10.3892 /$ ijmm.2018.3597

\begin{abstract}
The present study aimed to identify the association between microRNA (miRNA/miR)-31a-5p and the development of hypertension, and its potential molecular mechanism. Reverse transcription-quantitative polymerase chain reaction (RT-qPCR) and western blot analyses were performed to validate the candidate miRNA and genes involved in hypertension, following which an online miRNA database search, luciferase assay, and RT-qPCR and western blot analyses were performed to confirm the interaction between miR-31a-5p and TP53. A MTT assay and flow cytometric analysis were utilized to determine the effect of miR-31a-5p on cell growth and apoptosis. The results revealed that miR-31a-5p and TP53 were the candidate miRNA and gene regulating hypertension, and that TP53 was the virtual target gene of miR-31a-5p with a binding site located in the TP53 3' untranslated region ( $\left.3^{\prime} \mathrm{UTR}\right)$. It was confirmed by luciferase activity that miR-31a-5p markedly reduced the luciferase activity of the Luc-wild-type-TP53-3'UTR, whereas the mutated putative miR-31a-5p binding located on the TP53-3'UTR was found to eliminate such an inhibitory effect. miR-31a-5p had no effect on specificity protein 1, E2F transcription factor 2 or forkhead box P3 luciferase activity. Smooth muscle cells collected from spontaneously hypertensive rats treated with gold nano-particles containing anti-rno-miR-31a-5p exhibited a lower growth rate and a higher apoptotic rate. The results of the RT-qPCR and western blot analyses showed that miR-31a-5p negatively regulated the expression of TP53, and transfection with the hsa-miR-31a-5p mimic significantly promoted cell growth
\end{abstract}

Correspondence to: Dr Xiaojuan Lin, Department of Cardiology, The People's Hospital of Tongchuan, 12 Jiankang Road, Tongchuan, Shaanxi 727000, P.R. China

E-mail: rno31ap53@163.com

Key words: microRNA-31a-5p, primary hypertension, apoptosis, pulmonary artery smooth muscle cells, TP53 and inhibited cell apoptosis, whereas transfection with the anti-hsa-miR-31a-5p mimic significantly suppressed cell growth and induced cell apoptosis. Taken together, these findings indicated that miR-31a-5p is involved in hypertension via the accelerated proliferation of arterial smooth muscle cells and inhibition of apoptosis through targeting TP53.

\section{Introduction}

High blood pressure (HBP) is a risk factor for cardiovascular disorders, including chronic kidney disease, congestive heart failure, myocardial infarction and stroke, and is a leading contributor to rates of mortality and morbidity in the world. Substantial progress has been made in the treatment of HBP via the application of $\alpha$-adrenoreceptor antagonists, $\beta$-blockers, calcium channel blockers, renin-angiotensin system inhibitors and diuretics. Despite the availability of a variety of blood pressure drugs, a larger number of patients with HBP are unable to get their blood pressure under control. It was estimated that only $51.9 \%$ of patients with HBP in the United States had their blood pressure under control $(<140 / 90 \mathrm{~mm} \mathrm{Hg})$ between 2011 and 2012 (1).

As a basic composition of vascular walls, vascular smooth muscle cells (VSMCs) have a well-differentiated contractile phenotype, which is important to maintain vascular tone (2). The increased proliferation of VSMCs is associated with $\operatorname{HBP}(3,4)$. Vascular inflammation is considered to be involved in vascular remodeling in a variety of cardiovascular disorders, including atherosclerosis and HBP (5).

The tumor inhibitor protein p53 (protein product of TP53, also known as p53) is known to be involved in the development of restenosis and atherosclerosis, VSMC growth and cell death (6). A previous study reported that elevated nuclear translocation of p53 in cancer cells was observed in response to therapy with microtubule stabilizing agents (MTSAs) (7). It is widely known that the microtubule stabilization triggered by MTSA is the primary mechanism attributable to the elevation in the association of p53 with microtubules and its nuclear export, which is associated with the apoptotic pathway dependent 
on p53 (7). Due to the tumor inhibitory functions of p53, this protein has attracted attention from the pulmonary arterial hypertension community (8). Mizuno et al revealed that mice with p53 knockout formed more serious pulmonary hypertension in response to chronic hypoxia than wild-type mice (9).

As small and non-coding RNA molecules, microRNAs (miRNAs) consist of $\sim 22$ nucleotides and binding to their target mRNAs to suppress translation, which have a key regulatory role in eukaryotic genes, particularly in cell proliferation, differentiation and apoptosis (10). Often, miRNAs can bind to the 3' untranslated region (3'UTR) of the mRNAs of target genes in an imperfect or perfect complementary manner, leading to translational repression or mRNA degradation (11). Increasing data have revealed that dysregulated miRNAs are associated with cardiovascular disorders, including vascular atherosclerosis, heart failure and cardiac hypertrophy (12). Baseline gene expression levels of miRNA-26b, miRNA-499, miRNA-208b, miRNA-21, miRNA-133a and miRNA-1 have been determined in peripheral blood mononuclear cells (PBMCs), cells identified to be important in the pathophysiology of target organ injury (13). These miRNAs were selected as they have a different expression profile in HBP, and have been associated with heart and vascular remodeling (14). The expression of miRNAs in the PBMCs of patients has been investigated, as PBMCs are of important in the cardiovascular complications of HBP (15).

A previous study demonstrated the differential expression of miR-31a-5p in the smooth muscle cells collected from an animal model of primary hypertension, compared with the control, and it has been reported that dysregulated p53 is associated with the molecular mechanism of smooth muscle cell apoptosis (16-18). The present study performed a search on an online miRNA database and found that miR-31a-5p virtually targets p53. In the present study, miR-31a-5p was found to target p53, and the association of p53 and miR-31a-5p in the occurrence of primary hypertension was confirmed.

\section{Materials and methods}

Animals. All experiments were performed in 28 male adult spontaneously hypertensive rat (SHR; 16 rats) and normotensive Wistar-Kyoto (WKY; 12 rats) rats (15-16 weeks old, weighing $430 \pm 40 \mathrm{~g}$ ) following the institutional guidelines that comply with the recommendations in the Guide for the Care and Use of Laboratory Animals published by the US National Institutes of Health (8th edition, 2011). All procedures of experiments were approved by the Experimental Animal Care and Use Committee of China Medical University (Shenyang, China). All rats were housed at room temperature $\left(23 \pm 2^{\circ} \mathrm{C}\right)$ with a $12 \mathrm{~h}-12 \mathrm{~h}$ light/dark cycle, and were provided with a rodent chow diet and drinking water throughout the experiment. Gold nanoparticles (AuNPs) with miR-31a-5p were used for treatment of the rats.

Isolation and culture of pulmonary artery smooth muscle cells (PASMCs). The PASMCs were isolated from tissue samples derived from the rats; forceps were utilized to mince the tissue samples, and $4 \mathrm{mg} / \mathrm{ml}$ dispase (Sigma-Aldrich; EMD Millipore, Bedford, MA, USA) was used to digest the tissues for $30 \mathrm{~min}$ at $37^{\circ} \mathrm{C}$, and subjected to additional incubation for another $5 \mathrm{~h}$. A $40 \mu \mathrm{m}$ cell strainer (BD Falcon, Bedford, MA, USA) was utilized to filter the dissociated cell suspension. Centrifugation was performed for $15 \mathrm{~min}$ at $107.3 \mathrm{x}$ g at $4^{\circ} \mathrm{C}$ to yield the cells. DMEM with $10 \%$ fetal bovine serum (FBS; HyClone; GE Healthcare Life Sciences, Logan, UT, USA), $100 \mu \mathrm{g} / \mathrm{ml}$ streptomycin and $100 \mathrm{U} / \mathrm{ml}$ penicillin was utilized to incubate the cells under a humidified atmosphere with $5 \%$ $\mathrm{CO}_{2} / 95 \%$ air at $37^{\circ} \mathrm{C}$. The medium was replaced at 2 -day intervals until the cells were cloned. The cells at passage three were used in subsequent experiments.

RNA isolation and reverse transcription-quantitative polymerase chain reaction ( $R T-q P C R$ ) analysis. TRIzol (Invitrogen; Thermo Fisher Scientific, Inc., Waltham, MA, USA) was utilized to isolate total RNA from the tissue samples, and the mirVana $^{\mathrm{TM}}$ PARIS ${ }^{\mathrm{TM}}$ kit (Applied Biosystems; Thermo Fisher Scientific, Inc.) was utilized to extract total RNA from the PASMCs, based on the manufacturer's protocol. Subsequently, $8 \%$ denaturing polyacrylamide gels were utilized to monitor RNA integrity. TaqMan miRNA assays was performed to perform RT-qPCR analysis, and a high-capacity cDNA archive kit (Applied Biosystems; Thermo Fisher Scientific, Inc.) was utilized to reverse transcribe miRNA to cDNA according to the manufacturer's protocol. A NanoDrop instrument (NanoDrop; Thermo Fisher Scientific, Inc., Wilmington, DE, USA) was utilized to determine the concentrations of RNA, and U6 served as a control to normalize the expression of miR-31a-5p. SYBR-Green-based detection systems (Applied Biosystems; Thermo Fisher Scientific, Inc.) were utilized to perform the PCR amplifications on a GeneAmp PCR 9700 Thermocycler (Applied Biosystems; Thermo Fisher Scientific, Inc.) in accordance with the manufacturer's protocol, using Standard Taq Reaction Buffer (10X, $5 \mu \mathrm{l})$, dNTPs $(10 \mathrm{mM}, 1 \mu \mathrm{l})$, Forward Primer $(10 \mu \mathrm{M}, 1 \mu \mathrm{l})$, Reverse Primer $(10 \mu \mathrm{M}, 1 \mu \mathrm{l})$, Template DNA (10 ng), Taq DNA Polymerase $(0.25 \mu \mathrm{l})$ and nuclease-free water $(50 \mu \mathrm{l})$. The primer sequences used were as follows: RT primer, 5'GTCGTATCCAGTGCGTGTCGTGGAGTCGGC AATTGCACTGGATACGACCAGCTA-3'; forward, 5'-GGG AGGCAAGATGCTGGCA-3' and reverse, 5'-CAGTGCGTG TCGTGGAGT-3'. The thermocycling conditions were as follows: $95^{\circ} \mathrm{C}$ for $30 \mathrm{sec}, 55^{\circ} \mathrm{C}$ for $30-60 \mathrm{sec}, 72^{\circ} \mathrm{C}$ for $30-60 \mathrm{sec}$ for 30 cycles, and $72^{\circ} \mathrm{C}$ for $5 \mathrm{~min}$. Melting curve analysis was utilized to confirm the lack of primer dimers and specificity of amplification. The $2^{-\Delta \Delta \mathrm{Cq}}$ method (19) was utilized to analyze the expression of TP53 mRNA and miR-31a-5p. All experiments were run three times.

Cell culture and transfection. Dulbecco's modified Eagle's medium (DMEM; Invitrogen; Thermo Fisher Scientific, Inc.) containing $10 \%$ FBS, $100 \mathrm{mg} / \mathrm{ml}$ streptomycin and $100 \mathrm{U} / \mathrm{ml}$ penicillin was utilized to culture the PASMCs under a humidified atmosphere of $5 \% \mathrm{CO}_{2}$ at $37^{\circ} \mathrm{C}$. Lipofectamine 2000 (Thermo Fisher Scientific, Inc.) was utilized to perform transient transfections of miR-31a-5p mimic, miRNA control, and TP53 small interfering (si)RNA. The sequences were as follows: miR-31a-5p mimic, 5'-AGGCAAGAUGCUGGC AUAGCUG-3'; miRNA control, 5'-CAGCUAUGCCAGCAU ACUUGCCU-3'. The TP53 siRNA sequence was as follows: 5'-TTTTGGGACTTGAGGCATCTG-3'. All experiments were performed in triplicate. 
Cell proliferation assay. The PASMCs were seeded into 24-well plates at a final concentration of $2 \times 10^{3}$ cells per well. An MTT assay was performed to evaluate cell viability 24, 48 and $72 \mathrm{~h}$ post-MTT addition. An ELISA reader (ELX-800 type; Bio-Tek Instruments, Inc., Winooski, VT, USA) was used to measure the optical density of each well at $570 \mathrm{~nm}$ to quantify cell proliferation. Each experiment was repeated three times.

Luciferase assay. RT-PCR was performed to amplify the p53 3'UTR containing the putative or mutated binding site of miR-31a-5p. The thermocycling conditions were as follows: $25^{\circ} \mathrm{C}$ for $10 \mathrm{~min}, 42^{\circ} \mathrm{C}$ for $50 \mathrm{~min}, 70^{\circ} \mathrm{C}$ for $15 \mathrm{~min}$ and $37^{\circ} \mathrm{C}$ for $20 \mathrm{~min}$. The PCR products were then inserted into the hR-luc luciferase coding sequence downstream, which was located in the pmir-RB-REPORT ${ }^{\mathrm{TM}}$ vector (Guangzhou RiboBio Co., Ltd., Guangzhou, China) following standard protocol. Lipofectamine 2000 (Invitrogen; Thermo Fisher Scientific, Inc.) was utilized to co-transfect the cells with the luciferase constructs and miR-31a-5p mimic or negative control. At $48 \mathrm{~h}$ post-transfection, a Dual Luciferase Reporter Assay system (Promega Corporation, Madison, WI, USA) was utilized to measure the luciferase activity of Renilla and Firefly based on the manufacturer's protocol. The Renilla luciferase activity was normalized to Firefly luciferase activity. Three independent experiments were performed.

Western blot analysis. RIPA buffer (Sigma-Aldrich; EMD Millipore) was utilized to extract protein from the cells at $48 \mathrm{~h}$ post-transfection following the standard protocol. The lysates were centrifuged at $13,000 \mathrm{~g}$ for $15 \mathrm{~min}$ at $4^{\circ} \mathrm{C}$ to collect the upper supernatant. The bicinchoninic acid method was utilized to measure the protein concentration. SDS-polyacrylamide gels (12.5\%; Invitrogen; Thermo Fisher Scientific, Inc.) were utilized to electrophorese $30 \mu \mathrm{g}$ of the extracted protein, which were then blotted onto polyvinylidene difluoride membranes (EMD Millipore, Bedford), followed by blocking with $5 \%$ non-fat milk. Specific primary antibodies against p53 (cat. no. 9282T; 1:5,000; Cell Signaling Technology, Inc., Beverly, MA, USA) and against $\beta$-actin (cat. no. 4967S; 1:80,000, Sigma; EMD Millipore) were added for incubation with the membrane for $12 \mathrm{~h}$ at $4^{\circ} \mathrm{C}$, and TBST buffer was utilized to wash the membrane three times, following which corresponding horseradish peroxidase-labeled mouse IgG secondary antibody (1:1,000; cat. no. HAF007; R\&D Systems, Inc., Minneapolis, MN, USA) was added for incubation with the membrane for $60 \mathrm{~min}$ at $4^{\circ} \mathrm{C}$. The chemiluminescent reagents (GE Healthcare Life Sciences) and X-ray films (Denville Scientific, Holliston, MA, USA) were used to visualize the protein bands in accordance with the manufacturer's protocol. All assays were performed three times.

Analysis of apoptosis. At $48 \mathrm{~h}$ post-transfection, the PASMCs were harvested, and PBS was utilized to wash cells. A FITC-Annexin V/Propidium Iodide Apoptosis Detection kit (BestBio, Shanghai, China) was used to resuspend and stain the cells according to the manufacturer's protocol. Flow cytometry (BD FACSCanto II; BD Biosciences, San Jose, USA) was used to analyze cell apoptosis. Each experiment was performed three times.

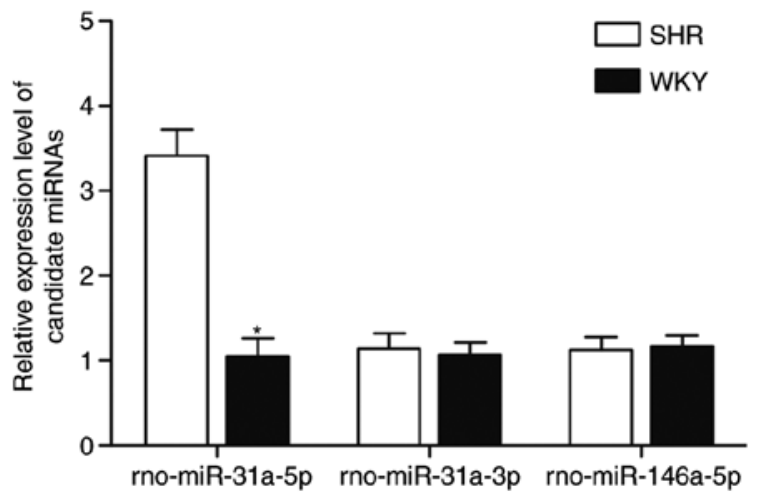

Figure 1. Reverse transcription-quantitative polymerase chain reaction analysis was used to confirmed candidate microRNAs involved in hypertension. Only the level of rno-miR-31a-5p was different between the SHR and WKY rats. miR, microRNA; SHR spontaneously hypertensive rat; WKY, Wistar-Kyoto. ${ }^{*} \mathrm{P}<0.05$ vs. the control.

Statistical analysis. The results are shown as the mean \pm standard deviation. Two-way analysis of variance or Student's t-test was used to perform all relative analyses using Statistical package for the Social Sciences for Windows Version 12.0 (SPSS, Inc., Chicago, IL, USA). $\mathrm{P}<0.05$ was considered to indicate a statistically significant difference. GraphPad Prism Version 5.0 (GraphPad Software, Inc., La Jolla, CA, USA) was used to generate all graphs.

\section{Results}

Identifying candidate miRNAs involved in hypertension. The SHR model is a genetic animal model for essential hypertension, which shows elevated blood pressure compared with normotensive WKY rats. RT-qPCR analysis was used to compare the expression of the candidate miRNAs (rno-miR-31a-5p, rno-miR-31a-3p and rno-miR-146a-5p) between the SHR and WKY rats, as shown in Fig. 1. Only rno-miR-31a-5p showed differential expression in the SHR rats in comparison with that in WKY rats, whereas no significant difference in levels of rno-miR-31a-3p and rno-miR-146a-5p were observed between the SHR and WKY rats.

Identifying candidate target genes of rno-miR-31a-5p. The PASMCs were collected from the SHR and WKY rats, and RT-qPCR analysis and western blot analysis were performed to examine candidate target genes, including p53, specificity protein 1 (SP1), E2F transcription factor 2 (E2F2) and forkhead box P3 (FOXP3), which were identified by searching the online miRNA database, (www.mirdb.org). The expression of these candidate target genes were determined and compared between cells collected from the SHR and WKY rats. As shown in Fig. 2, only the mRNA and protein (Fig. 2A) levels of p53 were decreased in the SHR group, compared with those in the WKY group, whereas the mRNA and protein levels of SP1 (Fig. 2B), E2F2 (Fig. 2C) and FOXP3 (Fig. 2D) were comparable between the SHR and WKY groups.

p53 is a candidate target gene of miR-31a-5p. To further validate p53 as a direct target of rno-miR-31a-5p, vectors 

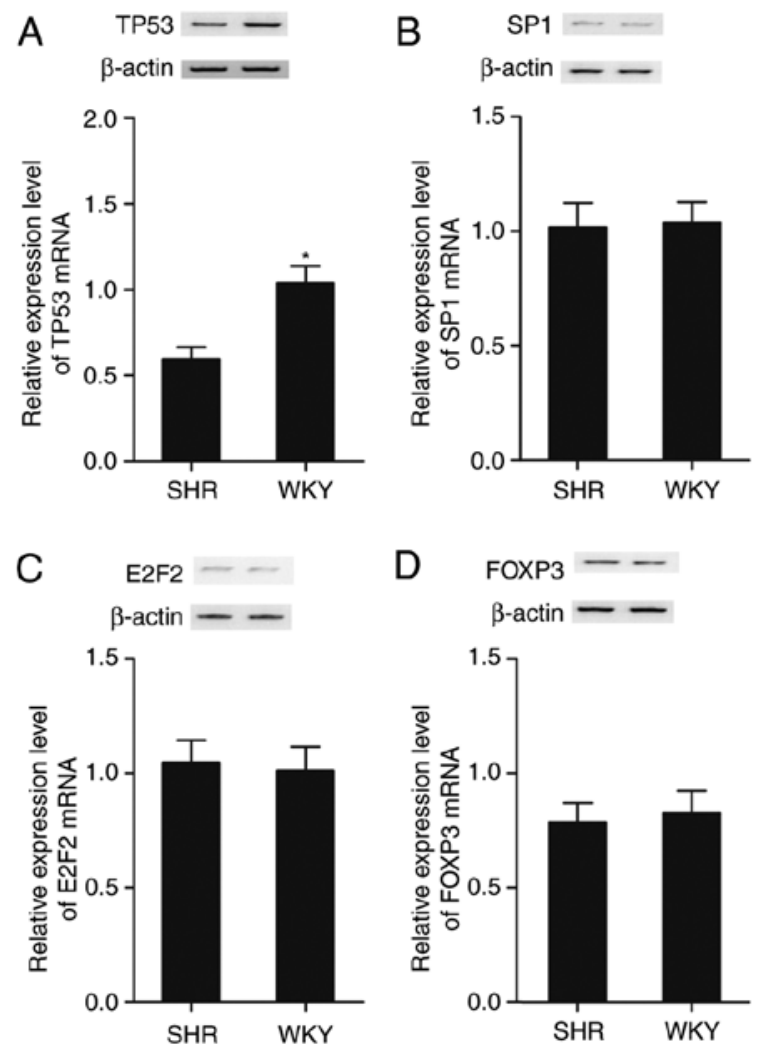

Figure 2. (A-D) Reverse transcription-quantitative polymerase chain reaction and western blot analyses were used to confirm candidate genes involved in hypertension. Only mRNA and protein levels of TP53 were significantly different between SHR and WKY rats. SP1, specificity protein 1; E2F2, E2F transcription factor 2; FOXP3, forkhead box P3; SHR spontaneously hypertensive rat; WKY, Wistar-Kyoto. ${ }^{*} \mathrm{P}<0.05$ vs. the control.

were constructed containing wild-type or mutant 3'UTR of candidate genes, including p53 (Fig. 3A), SP1 (Fig. 3B), E2F2 (Fig. 3C) and FOXP3 (Fig. 3D), all of which had a complementary binding site of miR-31a-5p located in their 3'UTR. Subsequently, a luciferase assay was performed by transfecting the rat PASMCs with the above-mentioned constructs together with rno-miR-31a-5p. As shown in Fig. 3A, miR-31a-5p markedly reduced the luciferase activity of the Luc-wild-p53-3'UTR relative to that in the control, whereas mutated putative miR-31a-5p binding located on p53-3'UTR was found to be refractile to this inhibitory effect. By contrast, there was no significant difference in the luciferase activity of either wild-type or mutant SP1 3'UTR (Fig. 3B), wild-type or mutant E2F2 3'UTR (Fig. 3C), or wild-type or mutant FOXP3 3'UTR (Fig. 3D) in the miR-31-5p-overexpressing cells, compared with that in the scramble control, confirming that miR-31-5p directly and negatively regulated the expression of $\mathrm{p} 53$, but not the expression of SP1, E2F2 or FOXP3.

Alteration of the expression of rno-miR-31a-5p affects the expression of 553 , and the proliferation and apoptosis of PASMCs of the SHR model. AuNPs containing scramble control or anti-rno-miR-31a-5p were used to treat the SHR rats, from which PASMCs were collected and the level of miR-31a-5p, expression of p53, and growth and apoptotic rates of the PASMCs were examined. As shown in Fig. 4A,
anti-rno-miR-31a-5p treatment suppressed the expression of rno-miR-31a-5p, compared with that in the scramble control. The downregulation of rno-miR-31a-5p upregulated the expression of p53 (Fig. 4B), and suppressed the proliferation of PASMCs (Fig. 4C) by promoting the apoptosis of the PASMCs (Fig. 4D and E), however, it did not affect the cell cycle status (Fig. 4F).

Alteration of has-miR-31a-5p affects the expression of p53, and proliferation and apoptosis of PASMCs human arterial smooth muscle cells. To further examine the role of miR-31-5p in the control of cell proliferation and apoptosis in PASMCs, the cells were transfected with the hsa-miR-31a-5p mimic, anti-hsa-miR-31a-5p mimic or the scramble controls. RT-qPCR analysis, western blot analysis, an MTT assay and flow cytometry were performed to determine the levels of miR-31-5p and $\mathrm{p} 53$, and the proliferation and apoptosis of the differently treated cells. As shown in Fig. 5, the anti-hsa-miR-31a-5p mimic (Fig. 5A) reduced the level of miR-31a-5p. The mRNA and protein levels of p53 in the cells were upregulated subsequent to transfect with the anti-hsa-miR-31a-5p mimic (Fig. 5B). The downregulation of miR-31a-5p by transfection with the anti-hsa-miR-31a-5p mimic inhibited the viability of arterial smooth muscle cells (Fig. 5C) and promoted the apoptosis of arterial smooth muscle cells (Fig. 5D and E), but did not affect the cell cycle status (Fig. 5F). The hsa-miR-31a-5p mimic (Fig. 6A) increased the level of miR-31a-5p. The mRNA and protein levels of p53 in the cells were downregulated following transfection with the hsa-miR-31a-5p mimic (Fig. 6B). The overexpression of miR-31a-5p significantly promoted the growth of the arterial smooth muscle cells (Fig. 6C) and significantly inhibited the apoptosis of the cells (Fig. 6D and E), but did not affect the cell cycle status (Fig. 6F). Collectively, these findings indicated that miR-31a-5p accelerated the proliferation of arterial smooth muscle cells and inhibited apoptosis via targeting p53.

\section{Discussion}

It has been shown that miRNAs, which modulate the proliferation or migration of endothelial progenitor cells and embryonic/mesenchymal stem cells (miR-702, miR-221/222 and miR-31), and reduce stem cell apoptosis and cancer (miR-31 and miR-702), are increased in hypertrophic RV, compared with those in controls according to unbiased quantitative $\mathrm{miR}$ microarray analysis $(20,21)$. It was previously revealed that only three miRs (miR-31a-5p, miR-31a-3p and miR-208b) were increased in the LV of PAH rats (22). The levels of C-kit and miR-31 were elevated in hypertrophic $\mathrm{RV}$ of PAH rats, compared with those in control rats, and miR-31 was elevated in the RV of PAH rats, which acts as a compensatory mechanism to decrease the reduction in capillary density, which is associated with the failing hearts (23). In the present study, candidate miRNAs (rno-miR-31a-5p, rno-miR-31a-3p and rno-miR-146a-5p) were investigated by comparing the expression levels between PASMCs from SHR and WKY rats, and it was revealed that only rno-miR-31a-5p was significantly upregulated in the SHR rats, compared with the WKY rats. Previously, studies have shown that miR-31 is involved in VSMC proliferation, angiogenesis and 

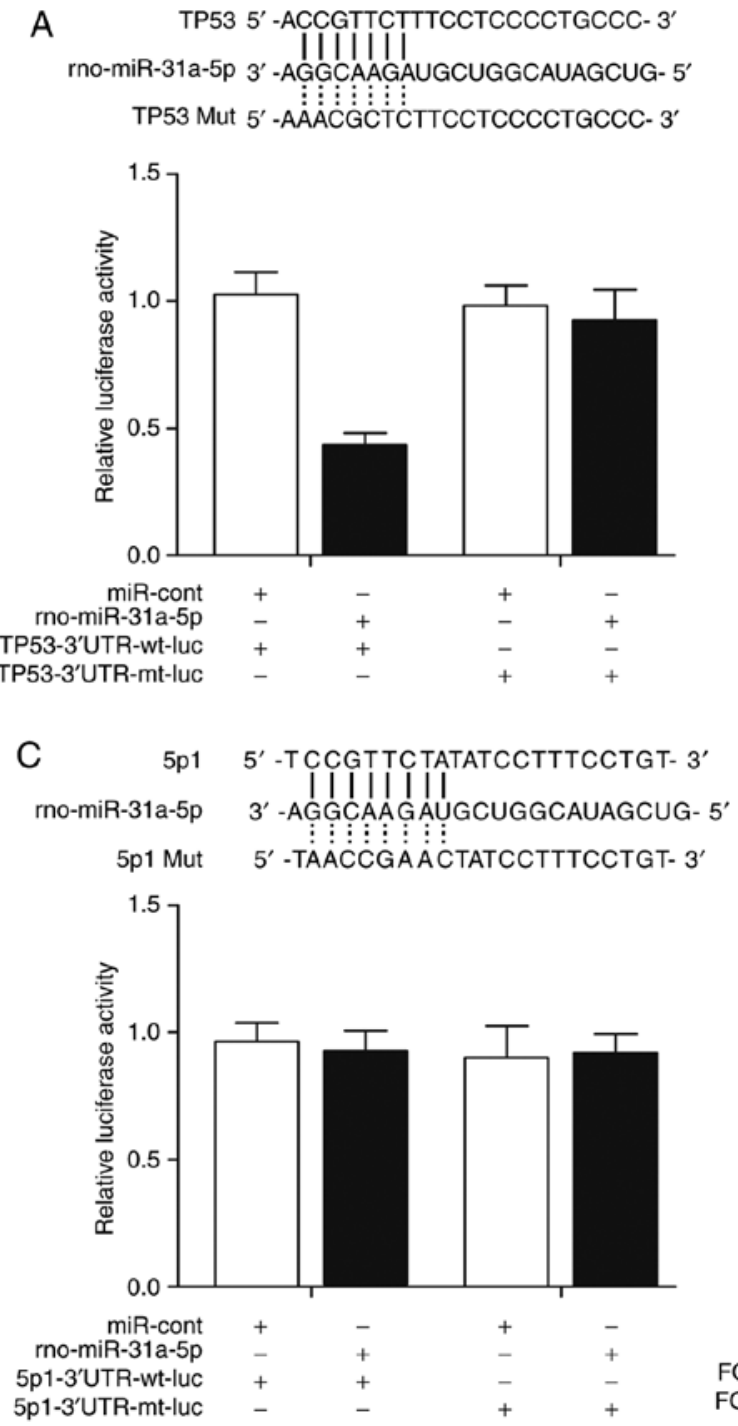
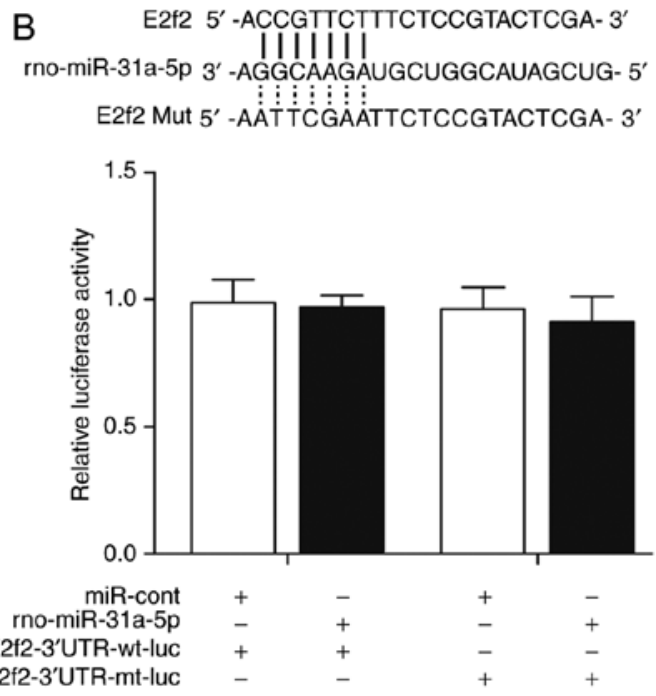

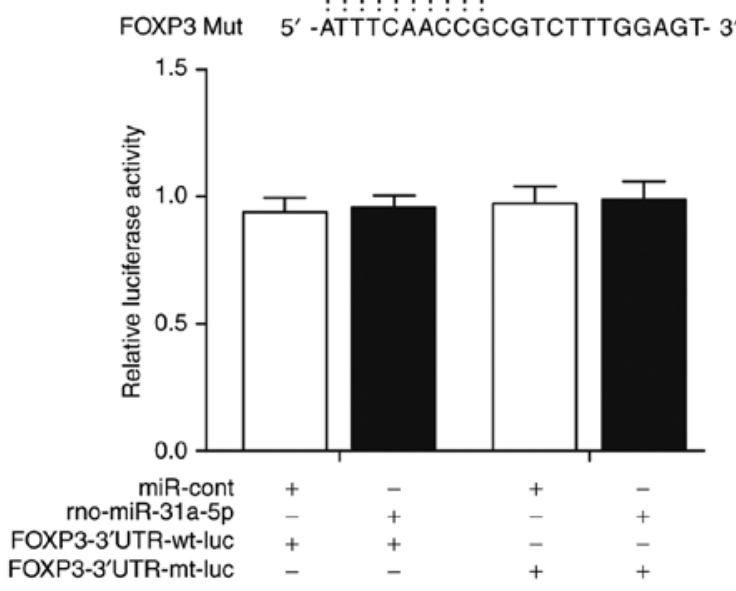

Figure 3. (A) Computational analysis showed that there were candidate seed sequences of miR-31a-5p in the 3'UTR of TP53. miR-31a-5p appeared to attenuate the luciferase activity of the wild-type TP53 3'UTR. (B) Computational analysis showed that there were candidate seed sequences of miR-31a-5p in the 3'UTR of SP1, and that miR-31a-5p had no effect on the luciferase activity of wild-type or mutant SP1 3'UTR. (C) Computational analysis showed that there were candidate seed sequences of miR-31a-5p in the 3'UTR of E2F2, and that miR-31a-5p had no effect on luciferase activity of wild-type or mutant E2F2 3'UTR. (D) Computational analysis showed that there were candidate seed sequences of miR-31a-5p in the 3'UTR of FOXP3, and that miR-31a-5p had no effect on luciferase activity of wild-type or mutant FOXP3 3'UTR. miR, microRNA; SP1, specificity protein 1; E2F2, E2F transcription factor 2; FOXP3, forkhead box P3; 3'UTR, 3' untranslated region; mut/mt, mutant; wt, wild-type; cont, control; luc, luciferase.

tumor metastasis, although miR-31 was found to be the most increased miRNA following acute myocardial infarction in rats (24-26). miR-31-induced cardioprotection was eliminated when the activation of nuclear factor (NF)- $\kappa \mathrm{B}$ was suppressed by $\mathrm{Adv}-\mathrm{dn} \mathrm{I} \kappa \mathrm{B} \alpha$ during the ischemia/reperfusion (I/R) process, revealing that diverse mechanisms may be involved in the miR-31/PKC $\varepsilon$ signaling-induced and I/R-induced activation of $\mathrm{NF}-\kappa \mathrm{B}$ in cardiac myocytes (27).

Patients with primary hypertension, a hereditary polygenic disease, eventually develop complications, including nephrosclerosis, cardiovascular remodeling and stroke. These complications are practical targets for the treatment of underlying HBP with blood pressure drugs. SHR rats, a genetic animal model for underlying hypertension, indicates poorer growth of cardiovascular organs compared with normotensive WKY rats (28). SHR-derived VSMCs in culture exhibit accelerated entry into the $\mathrm{S}$ phase of the cell cycle, aberrant contact suppression, a higher specific growth rate, and nonspecific hyperproliferation responding to a variety of growth factors, compared with cells from WKY rats (29). In the present study, candidate genes of miR-31a-5p were investigated using an online miRNA database, and four genes were found, including p53, SP1, E2F2 and FOXP3, which may be target genes of miR-31a-5p with a complementary binding site of miR-31a-5p located in their 3'UTR respectively. It was then found that only the mRNA and protein levels of p53 were differential in the SHR group, compared with those in the WKY group, whereas the mRNA and protein levels of SP1, E2F2 and FOXP3 in the SHR group did not differ significantly compared with those in the WKY group, and this regulatory association was further confirmed by the results of the luciferase assay.

p53, a tumor inhibitor, is a key transcription factor that modulates several cellular processes. It can suppress cell proliferation by triggering cell cycle arrest in the G1, G2 and S 
A

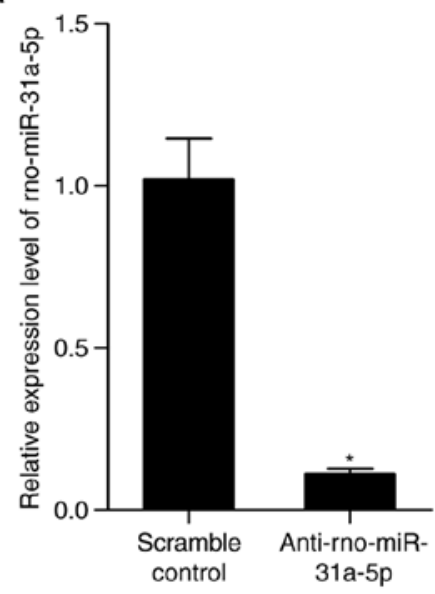

D

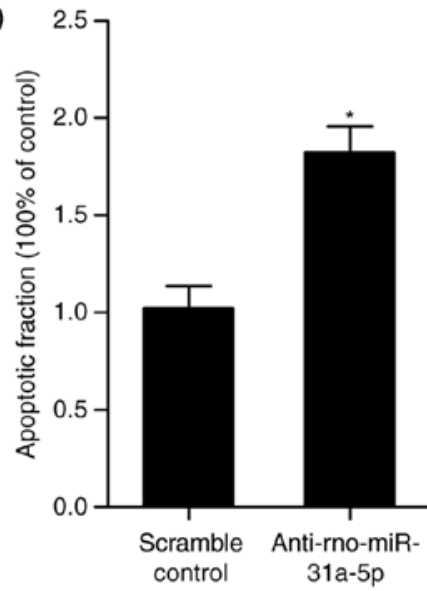

B
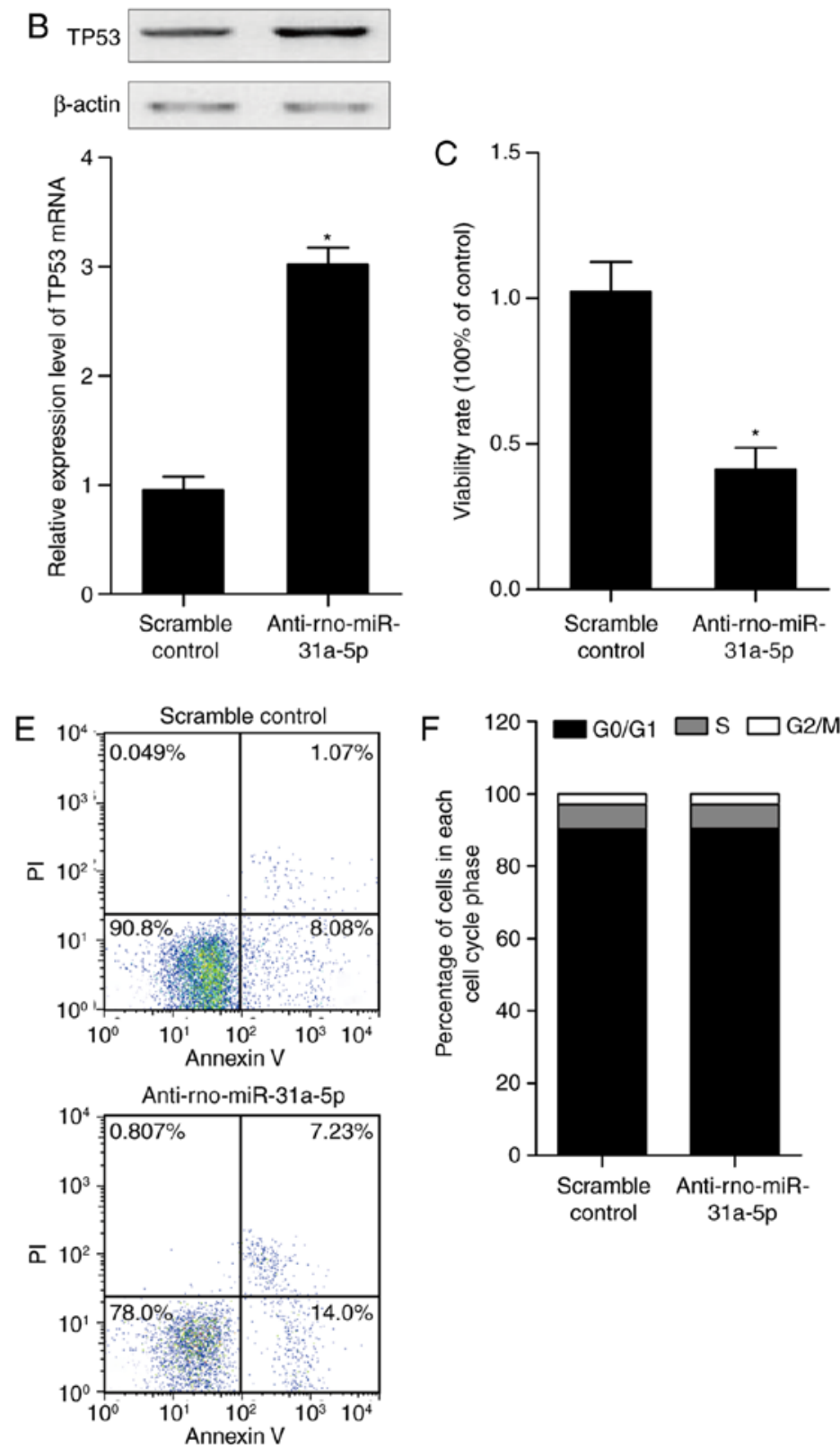

Figure 4. Arterial smooth muscle cells from spontaneously hypertensive rats treated with gold nanoparticles containing scramble control or anti-rno-miR-31a-5p mimic were collected. (A) Anti-rno-miR-31a-5p inhibited the expression of miR-31a-5p. (B) Anti-rno-miR-31a-5p increased the expression of TP53. (C) Anti-rno-miR-31a-5p downregulated cell viability. (D) Anti-rno-miR-31a-5p induced cell apoptosis. (E) Representative flow cytometry plot for apoptosis status. (F) Treatment did not affect the cell cycle status of the cells. miR, microRNA.

phases of the cell cycle (30); its expression is associated with elevated cell apoptosis in vitro and in vivo (31). p53 predominantly acts as a transcription factor, which can induce various anti-proliferative programs by the inhibition or activation of critical genes or effects (32). For VSMCs, the downregulation of p53 occurs prior to VSMC migration and proliferation (33). A hypermethylation status has been observed in $\mathrm{p} 53$ promoter region when treatment by Hcy, which indicates a causative function for VSMC proliferation (17). Therefore, p53 is considered a potent negative modulator of cell proliferation, including that of VSMCs. The p53 tumor inhibitor protein is stimulated in response to various cellular stresses, including nucleotide depletion, oncogene activation and DNA damage, with $\mathrm{p} 21$ and MDM2 being the most well-known examples of these targets (34). Indirect p53-mediated suppression is also involved via stimulation of its direct transcriptional target, known as p21 (35). Representing the Ink4a/Cip1 family of cyclin-dependent kinase (CDK) inhibitors, p21 suppressors bind to and suppress CDK4 and CDK6/cyclin D complexes to trigger cell-cycle arrest, leading to the activation and de-phosphorylation of retinoblastoma pocket proteins, which act together with E2F transcription factors to inhibit genes associated with the cell cycle, including hTERT, EZH2 and CHK1, which are p53-inhibition targets modulated by p21 (36,37). In the present study, PASMCs were collected from SHR rats treated with AuNPs containing scramble control or anti-rno-miR-31a-5p mimic, and it was revealed that anti-rno-miR-31a-5p suppressed the expression of miR-31a-5p, compared with the scramble control, and attenuated miR-31a-5p-inhibited PASMC growth, but markedly increased 

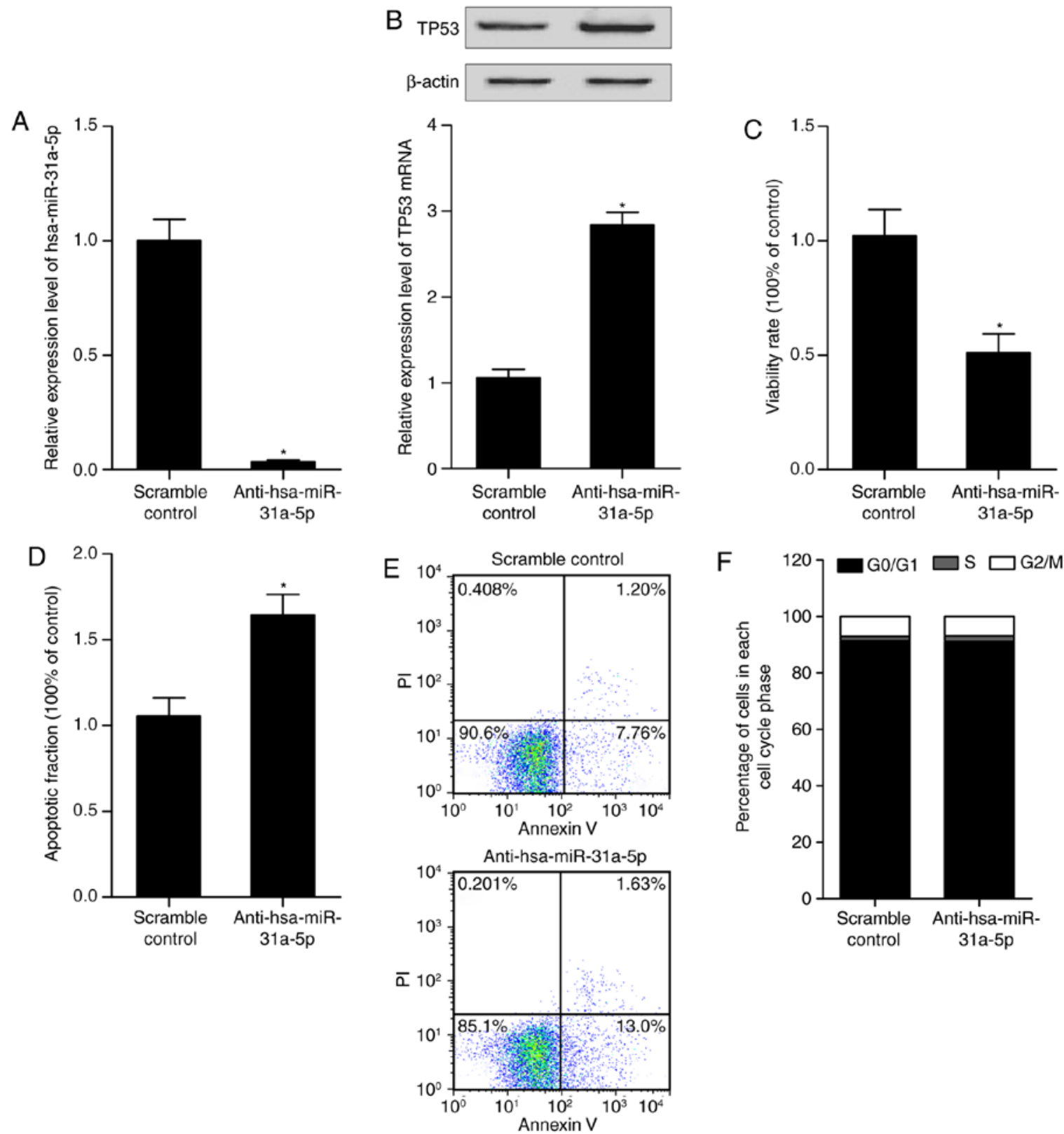

Figure 5. Reverse transcription-quantitative polymerase chain reaction and western blot analyses, an MTT assay and flow cytometry were performed to determine the levels of miR-31-5p and TP53, and the proliferation and apoptosis of cells transfected with anti-hsa-miR-31a-5p mimic. (A) Anti-rno-miR-31a-5p inhibited the expression of miR-31a-5p. (B) Anti-rno-miR-31a-5p increased the expression of TP53. (C) Anti-rno-miR-31a-5p downregulated cell viability. (D) Anti-rno-miR-31a-5p induced cell apoptosis. (E) Representative flow cytometry plot for apoptosis status. (F) Treatment did not affect the cell cycle status of the cells. miR, microRNA.

apoptosis of the PASMCs. Finally, RT-qPCR analysis, western blot analysis, an MTT assay, and flow cytometry were used to determine the levels of miR-31-5p and p53, and the proliferation and apoptosis of cells transfected with the hsa-miR-31a-5p mimic and anti-hsa-miR-31a-5p mimic. It was revealed that miR-31a-5p markedly suppressed the expression of p53, and miR-31a-5p accelerated the proliferation of PASMCs but inhibited apoptosis.

The effects of miR-31 have been associated with the status of p53. miR-31 acts as an inhibitor only in tumor cells that harbor mutant p53, which indicates miR-31 as a target for therapy in patients with p53-deficient tumors (38). Of note, the p53 mutation is an early indicator in esophageal squamous cell cancer (ESCC); additionally, alterations in the p53 status may contribute to context-dependent effects of several molecules, which include microRNAs, including miR-31 $(38,39)$. It has been shown that the inhibitory role of miR-31 in ESCC relies on a deficiency of p21 in addition to modulation by p53 (40).

In conclusion, the findings of the present study demonstrated that the deregulation of miR-31a-5p was associated with the risk of hypertension by suppressing the apoptosis of arterial smooth muscle cells. It was found that p53, a well-known tumor suppressor, was a direct target gene of miR-31a-5p, which was important in the apoptosis of arterial smooth muscle cells and was involved in the pathogenesis of hypertension. Therefore, miR-31a-5p may be a novel therapeutic strategy for the treatment of hypertension. 

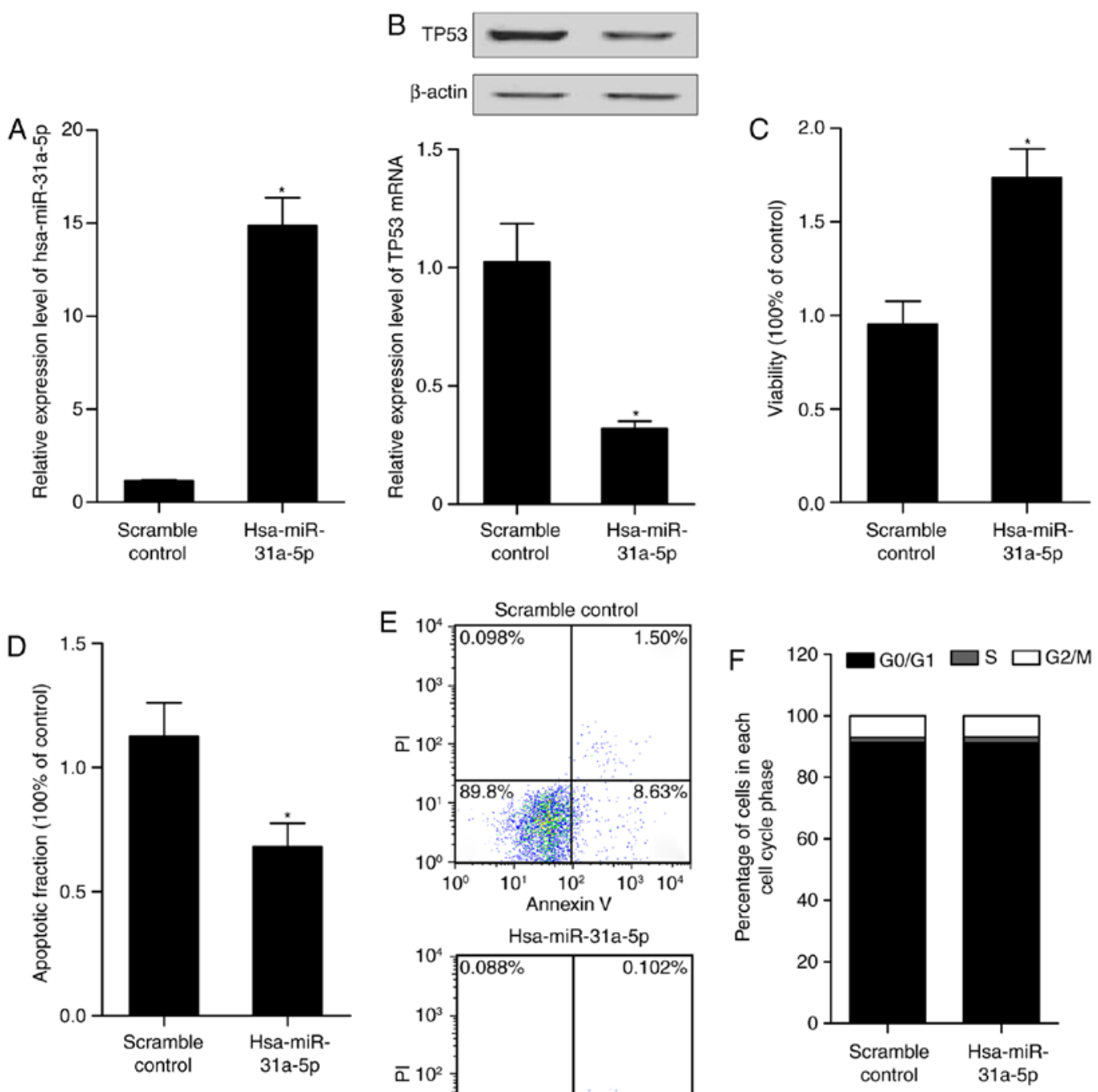

Figure 6. Reverse transcription-quantitative polymerase chain reaction and western blot analyses, an MTT assay and flow cytometry were performed to determine the levels of miR-31-5p and TP53, and the proliferation and apoptosis of cells transfected with hsa-miR-31a-5p mimic. (A) Rno-miR-31a-5p increased the expression of miR-31a-5p. (B) Rno-miR-31a-5p decreased the expression of TP53. (C) Rno-miR-31a-5p increased cell viability. (D) Rno-miR-31a-5p protected cells from apoptosis. (E) Representative flow cytometry plot for apoptotic status. (F) Treatment did not affect the cell cycle status of the cells. miR, microRNA.

\section{Funding}

No funding was received.

\section{Availability of data and materials}

The datasets generated and analyzed in the present study are included in this published article.

\section{Authors' contributions}

QF: Study planning, data collection, data analysis and interpretation, preparation of the manuscript and literature analysis. TT: Study planning, data collection, data analysis and interpretation and literature analysis. JL: Study planning, data collection, data analysis and interpretation and literature analysis. LZ: Data collection, data analysis and interpretation and literature analysis. JQ: Data analysis and interpretation, preparation of the manuscript and literature analysis. XL: Data collection, data analysis and interpretation and funds collection.

\section{Ethics and consent to participate}

All procedures of experiments were approved by the Experimental Animal Care and Use Committee of China Medical University (Shenyang, China).

\section{Consent for publication}

Not applicable.

\section{Competing interests}

The authors declare that they have no competing interests. 


\section{References}

1. Nwankwo T, Yoon SS, Burt V and Gu Q: Hypertension among adults in the United States: National Health and Nutrition Examination Survey, 2011-2012. NCHS Data Brief: 1-8, 2013.

2. Chiong M, Cartes-Saavedra B, Norambuena-Soto I, Mondaca-Ruff D, Morales PE, Garcia-Miguel M and Mellado R: Mitochondrial metabolism and the control of vascular smooth muscle cell proliferation. Front Cell Dev Biol 2: 72, 2014.

3. Ragolia L, Palaia T, Paric E and Maesaka JK: Prostaglandin D2 synthase inhibits the exaggerated growth phenotype of spontaneously hypertensive rat vascular smooth muscle cells. J Biol Chem 278: 22175-22181, 2003

4. Zheng GQ, Zhang GH, Xu XW, Wang JW, Yu LB, Zhang YN, Huang JW, Li YL, Braudt-Rauf PW and Xia ZL: Association of telomere length with chromosomal damage among Chinese workers exposed to Vinyl Chloride monomer. J Occup Environ Med 59: e252-e256, 2017

5. Pacurari M, Kafoury R, Tchounwou PB and Ndebele K: The Renin-Angiotensin-aldosterone system in vascular inflammation and remodeling. Int J Inflam 2014: 689360, 2014

6. Mercer J, Figg N, Stoneman V, Braganza D and Bennett MR: Endogenous p53 protects vascular smooth muscle cells from apoptosis and reduces atherosclerosis in ApoE knockout mice. Circ Res 96: 667-674, 2005.

7. Giannakakou P, Sackett DL, Ward Y, Webster KR Blagosklonny MV and Fojo T: p53 is associated with cellular microtubules and is transported to the nucleus by dynein. Nat Cell Biol 2:709-717, 2000.

8. Krones-Herzig A, Adamson E and Mercola D: Early growth response 1 protein, an upstream gatekeeper of the p53 tumor suppressor, controls replicative senescence. Proc Natl Acad Sci USA 100: 3233-3238, 2003.

9. Mizuno S, Bogaard HJ, Kraskauskas D, Alhussaini A, Gomez-Arroyo J, Voelkel NF and Ishizaki T: p53 Gene deficiency promotes hypoxia-induced pulmonary hypertension and vascular remodeling in mice. Am J Physiol Lung Cell Mol Physiol 300: L753-L761, 2011.

10. Sempere LF, Freemantle S, Pitha-Rowe I, Moss E, Dmitrovsky E and Ambros V: Expression profiling of mammalian microRNAs uncovers a subset of brain-expressed microRNAs with possible roles in murine and human neuronal differentiation. Genome Biol 5 R13, 2004.

11. Ambros V: The functions of animal microRNAs. Nature 431: 350-355, 2004

12. Pan ZW, Lu YJ and Yang BF: MicroRNAs: A novel class of potential therapeutic targets for cardiovascular diseases. Acta Pharmacol Sin 31: 1-9, 2010.

13. Zapolska-Downar D, Siennicka A, Chelstowski K, Widecka K, Goracy I, Halasa M, Machalinski B and Naruszewicz M: Is there an association between angiotensin-converting enzyme gene polymorphism and functional activation of monocytes and macrophage in young patients with essential hypertension? J Hypertens 24: 1565-1573, 2006.

14. Kontaraki JE, Marketou ME, Parthenakis FI, Maragkoudakis S, Zacharis EA, Petousis S, Kochiadakis GE and Vardas PE: Hypertrophic and antihypertrophic microR NA levels in peripheral blood mononuclear cells and their relationship to left ventricular hypertrophy in patients with essential hypertension. J Am Soc Hypertens 9: 802-810, 2015.

15. Muller DN, Kvakan H and Luft FC: Immune-related effects in hypertension and target-organ damage. Curr Opin Nephrol Hypertens 20: 113-117, 2011

16. Palao T, Sward K, Jongejan A, Moerland PD, de Vos J, van Weert A, Arribas SM, Groma G, vanBavel E and Bakker EN: Gene expression and microrna expression analysis in small arteries of spontaneously hypertensive rats. Evidence for ER stress. PLoS One 10: e0137027, 2015.

17. Jacquin S, Rincheval V, Mignotte B, Richard S, Humbert M, Mercier O, Londono-Vallejo A, Fadel E and Eddahibi S: Inactivation of p53 is sufficient to induce development of pulmonary hypertension in rats. PLoS One 10: e0131940, 2015.

18. Cai L, Li J, Zhang X, Lu Y, Wang J, Lyu X, Chen Y, Liu J, Cai H, Wang Y, et al: Gold nano-particles (AuNPs) carrying anti-EBV-miR-BART7-3p inhibit growth of EBV-positive nasopharyngeal carcinoma. Oncotarget 6: 7838-7850, 2015.

19. Livak KJ and Schmittgen TD: Analysis of relative gene expression data using real-time quantitative PCR and the 2(-Delta Delta C(T)) method. Methods 25: 402-408, 2001.
20. Kim BM and Choi MY: Non-canonical microRNAs miR-320 and miR-702 promote proliferation in Dgcr8-deficient embryonic stem cells. Biochem Biophys Res Commun 426: 183-189, 2012.

21. Dong Z, Zhong Z, Yang L, Wang S and Gong Z: MicroRNA-31 inhibits cisplatin-induced apoptosis in non-small cell lung cancer cells by regulating the drug transporter ABCB9. Cancer Lett 343 . 249-257, 2014.

22. Joshi SR, Dhagia V, Gairhe S, Edwards JG, McMurtry IF and Gupte SA: MicroRNA-140 is elevated and mitofusin-1 is downregulated in the right ventricle of the Sugen5416/hypoxia/normoxia model of pulmonary arterial hypertension. Am J Physiol Heart Circ Physiol 311: H689-H698, 2016.

23. Alzoubi A, Toba M, Abe K, O'Neill KD, Rocic P, Fagan KA, McMurtry IF and Oka M: Dehydroepiandrosterone restores right ventricular structure and function in rats with severe pulmonary arterial hypertension. Am J Physiol Heart Circ Physiol 304: H1708-H1718, 2013.

24. Liu X, Cheng Y, Chen X, Yang J, Xu L and Zhang C: MicroRNA-31 regulated by the extracellular regulated kinase is involved in vascular smooth muscle cell growth via large tumor suppressor homolog 2. J Biol Chem 286: 42371-42380, 2011.

25. Pedrioli DM, Karpanen T, Dabouras V, Jurisic G, van de Hoek G, Shin JW, Marino D, Kalin RE, Leidel S, Cinelli P, et al: MiR-31 functions as a negative regulator of lymphatic vascular lineage-specific differentiation in vitro and vascular development in vivo. Mol Cell Biol 30: 3620-3634, 2010.

26. Shi B, Guo Y, Wang J and Gao W: Altered expression of microRNAs in the myocardium of rats with acute myocardial infarction. BMC Cardiovasc Disord 10: 11, 2010.

27. Wang Y, Men M, Yang W, Zheng H and Xue S: MiR-31 downregulation protects against cardiac ischemia/reperfusion injury by targeting protein kinase $\mathrm{C}$ epsilon (PKCepsilon) directly. Cell Physiol Biochem 36: 179-190, 2015.

28. Huang CY, Kuo CH, Pai PY, Ho TJ, Lin YM, Chen RJ, Tsai FJ, Vijaya Padma V, Kuo WW and Huang CY: Inhibition of HSF2 SUMOylation via MEL18 upregulates IGF-IIR and leads to hypertension-induced cardiac hypertrophy. Int J Cardiol S0167-5273: 33565-33569, 2017

29. Ueno T, Takagi H, Fukuda N, Takahashi A, Yao EH, Mitsumata M, Hiraoka-Yamamoto J, Ikeda K, Matsumoto K and Yamori Y: Cardiovascular remodeling and metabolic abnormalities in SHRSP.Z-Lepr(fa)/IzmDmcr rats as a new model of metabolic syndrome. Hypertens Res 31: 1021-1031, 2008.

30. Cannell IG, Merrick KA, Morandell S, Zhu CQ, Braun CJ, Grant RA, Cameron ER, Tsao MS, Hemann MT and Yaffe MB: A pleiotropic Rna-binding protein controls distinct cell cycle checkpoints to drive resistance of p53-defective tumors to chemotherapy. Cancer Cell 28: 623-637, 2015.

31. Muller P, Ceskova P and Vojtesek B: Hsp90 is essential for restoring cellular functions of temperature-sensitive $\mathrm{p} 53$ mutant protein but not for stabilization and activation of wild-type p53: Implications for cancer therapy. J Biol Chem 280: 6682-6691, 2005.

32. Zilfou JT and Lowe SW: Tumor suppressive functions of p53. Cold Spring Harb Perspect Biol 1: a001883, 2009.

33. Rodriguez-Campos A, Ruiz-Enriquez P, Faraudo S and Badimon L: Mitogen-induced p53 downregulation precedes vascular smooth muscle cell migration from healthy tunica media and proliferation. Arterioscler Thromb Vasc Biol 21: 214-219, 2001.

34. Vousden KH and Prives C: Blinded by the light: The growing complexity of p53. Cell 137: 413-431, 2009.

35. Laptenko $\mathrm{O}$ and Prives $\mathrm{C}$ : Transcriptional regulation by $\mathrm{p} 53$ : One protein, many possibilities. Cell Death Differ 13:951-961, 2006

36. Tang X, Milyavsky M, Shats I, Erez N, Goldfinger N and Rotter V: Activated p53 suppresses the histone methyltransferase EZH2 gene. Oncogene 23: 5759-5769, 2004.

37. Carcagno AL, Marazita MC, Ogara MF, Ceruti JM, Sonzogni SV, Scassa ME, Giono LE and Canepa ET: E2F1-mediated upregulation of p19INK4d determines its periodic expression during cell cycle and regulates cellular proliferation. PLoS One 6: e21938,2011.

38. Creighton CJ, Fountain MD, Yu Z, Nagaraja AK, Zhu H, Khan M, Olokpa E, Zariff A, Gunaratne PH, Matzuk MM, et al: Molecular profiling uncovers a p53-associated role for microRNA-31 in inhibiting the proliferation of serous ovarian carcinomas and other cancers. Cancer Res 70: 1906-1915, 2010

39. Yang Y, Tarapore RS, Jarmel MH, Tetreault MP and Katz JP: p53 mutation alters the effect of the esophageal tumor suppressor KLF5 on keratinocyte proliferation. Cell Cycle 11: 4033-4039, 2012.

40. Ning Z, Zhu H, Li F, Liu Q, Liu G, Tan T, Zhang B, Chen S, Li G, Huang D, et al: Tumor suppression by miR-31 in esophageal carcinoma is p21-dependent. Genes Cancer 5: 436-444, 2014

This work is licensed under a Creative Commons Attribution-NonCommercial-NoDerivatives 4.0 International (CC BY-NC-ND 4.0) License. 